\title{
La nueva era de la polarización de los hábitos de consumo: Lujo Vs. Low- cost
}

Celia Rangel Pérez ${ }^{1}$ | celia.rangel@esic.edu

ESIC BUSINESS \& MARKETING SCHOOL

Resumen: En este artículo se estudian los efectos de las marcas low-cost y de las marcas de lujo en los hábitos de consumo en la sociedad occidental. Por un lado, existe un grupo de consumidores que desea emplear su dinero del mejor modo y por otro lado, existe otro grupo de la sociedad que demanda productos y servicios cada vez más personalizados y exclusivos. Este fenómeno está provocando un cambio en la percepción colectiva en torno al precio, obligando a las marcas a reforzar su posicionamiento y su propuesta de valor para fortalecer la lealtad hacia la marca y así mantener la rentabilidad de la compañía. Pero pese a lo que pueda parecer, las marcas de lujo y low-cost comparten planteamientos estratégicos: ambas se esfuerzan en dar valor añadido para desbancar a la competencia y en poner en el centro de su estrategia al consumidor para construir el universo de su marca.

Palabras clave: Lujo, low-cost, hábitos de consumo, polarización.

Abstract: This article studies the impact of low-cost brands and luxury brands in consumer habits in the Western society. On the one hand, there is a group of consumers who want to use their money the best way they can, and on the other hand, there is another group that demands personalized and exclusive products and services. This phenomenon is caused by a change in price perception, making brands reinforce their positioning and value proposition to strengthen client loyalty and maintain business profit figures. Contrary to what one might think, luxury brands and low-cost ones share strategic approaches: both try to add value to oust the competition and place the consumer in a central position so as to build their brand universe.

Key words: Luxury, low-cost, consumer habits, polarization.

1 Celia Rangel es Doctora y Licenciada en Publicidad y Relaciones Públicas por la Universidad Complutense de Madrid. Celia es profesora del Departamento de Publicidad en ESIC Business \& Marketing School desde 2011 en el área de Grado y Postgrado. Actualmente, Celia combina su labor docente con su actividad de consultoría en Morillas Branding Agency 


\section{Introducción}

En un entorno de económico mundial todavía recuperándose de la recesión llama la atención que, según Forbes (2014), las personas ricas cada vez tienen más ingresos y por el contrario, según Naciones Unidas (2014), más de 1.000 millones de personas subsisten con menos de un dólar al día. Una de las consecuencias de la crisis que la mayoría de expertos señala es un cambio en la forma de consumir y por ende, de valorar lo que se compra y cómo se usa. Se habla de una sociedad bipolar provocada, según sostienen Massimo Gaggi y Edoardo Narduzzi (2006), por una reducción de la clase media. En palabras de dichos autores, "por todas partes aparecen nuevos ricos que ostentan su opulencia; entre los trabajadores (en general, los no especializados) y pensionistas, se detectan focos de pobreza imprevistos; la clase media, en progresivo decrecimiento, pierde renta y seguridad" (2006: IX). De hecho, se puede distinguir entre la "sociedad creativa" que sabe asegurarse una renta y que está formada por "profesionales, operadores de mercados financieros, trabajadores del conocimiento, empleados de servicios 'protegidos' o empresarios de sectores innovadores." Y por otro lado, se encuentra "la fuerza-trabajo de baja especialización" compuesta por "obreros de la industria expuesta a la competencia internacional y empleados de los servicios tradicionales [desde el transporte a la restauración]" que "se encuentran comprimidos entre [la] reducción de rentas y [la] reducción de garantías sociales" (2006: 29 - 30). La radicalización del consumo que sostienen Gaggi y Narduzzi se puede comprobar, por ejemplo, en el éxito de ventas de Dacia Logan en Europa (2007), la marca de bajo coste de Renault, que originariamente estaba pensada para ser comercializada en países emergentes. Por otra parte, la industria del lujo sigue creciendo (Benito, 2014) en momentos de recesión, sobre todo, gracias al incremento del gasto en el mercado asiático.

Para estudiar las consecuencias de una sociedad bipolar caracterizada por una radicalización del consumo, en este artículo se plantean los siguientes objetivos:

- Analizar los cambios en la percepción del factor precio de los consumidores.

- Estudiar los efectos de las marcas low-cost y de las marcas de lujo en los hábitos de consumo en los consumidores.

- Establecer las similitudes y diferencias estratégicas de las marcas de lujo y low-cost

Para responder a los objetivos planteados en este trabajo, se realiza una reflexión e investigación crítica de la literatura actual sobre la percepción del factor precio, el fenómeno del low-cost, las marcas de lujo, los diferentes tipos de consumidores de marcas de lujo y low-cost y las diferentes estrategias que siguen tanto las empresas de productos y servicios low-cost como las de lujo. 


\section{La sociedad bipolar}

La polarización del consumo (Gaggi y Narduzzi, 2006) es fruto de la recesión económica que ha provocado que el precio sea un factor muy importante en la compra. Se prevé que "los clientes van a mantener por mucho tiempo la alta sensibilidad al precio, por lo que [se] deberá trasladar cada vez con mayor claridad la información adecuada del posicionamiento de cada compañía y, por tanto, del contenido de calidad que ofrece" (Valls et al., 2008: 79). Los elementos que inciden en la sensibilidad al precio son los "atribuibles a la estrategia de producto o servicio, como el valor, la calidad, la marca. Los atributos físicos, los intangibles y los psicológicos. Los elementos atribuibles al consumidor, como la búsqueda de la reducción del riesgo y la lealtad" (Valls et al., 2008: 79). En consecuencia, conviene establecer los precios en función de la percepción del cliente. Valls y Alfaro señalan los siguientes pasos a la hora de fijar precios basados en dicha percepción:

[En primer lugar, conviene] identificar (...) una perspectiva más comportamental, basada en la relación calidad/precio, donde existe intercambio de actividades [tangibles o intangibles] y además recompensas/costes. [A partir de ahí, se debe] comprender cuál es el valor sostenible que el producto o servicio aporta al cliente [para poder] crear el valor [y comunicarlo] a partir de elementos tangibles e intangibles. [De este modo, se podrá] convencer a los clientes de lo que vale el valor ofrecido y de lo que deben pagar. [Por último, se trata de] medir el valor y traducirlo a precio (2008: 27).

Gaggi y Narduzzi destacan (2006) que el fin de la clase media está avivando una sociedad "que ya no tiene obreros, pero tampoco un papel preciso para profesores y médicos: una realidad indiferenciada -más monocorde que homogénea- cada vez menos capaz de rechazar la diversidad de aspiraciones, de las necesidades, de los deseos de consumo" (2006: 6). La consecuencia, según Gaggi y Narduzzi, es una nueva "clase de masas" que:

Se caracteriza por un consumo de bajo coste: adquisiciones nómadas fácilmente repetibles y reconocibles en todo el mundo: Ikea, Ryanair, Wal-Mart, Virgin, Zara, Prêt à Manger, H\&M son sólo algunas de las marcas que interpretan la nueva identidad comportamental del fin de la clase media (2007: 7).

Josep-Francesc Valls y Manuel Alfaro (2008) consideran que las marcas low-cost nacieron en el sector de los vuelos domésticos y se extendieron a las compañías de gran consumo para llegar después al resto de categorías del mercado. En palabras de los autores:

El fenómeno low cost aparece a finales de la década de 1990, con el nacimiento de las compañías aéreas de bajo coste. Tiene dos precedentes: el primero, a finales de la década de 1970, en Estados Unidos, cuando se produce la liberación aérea; y el segundo, a principios de los años noventa cuando una serie de empresas siguieron la estela de Marlboro y Procter \& Gamble, impulsando la fijación de precios bajos diarios. La práctica seguida por Wal-Mart, entre otros, consistía en convertir la competencia de precios en un elemento fundamental de la estrategia de marketing, que debía ser seguida por la cadena de distribución. Las alianzas entre los fabricantes y los distribuidores permitieron una importante reducción de precios al contado, no sólo de forma temporal. El resultado fue que los consumidores se encontraron con precios bajos diarios (...) El fenómeno low cost está modificando la mentalidad colectiva en torno al precio. En una primera fase se extendió rápidamente a través de gran parte de los subsectores del turismo y el ocio, hoteles, parques temáticos, restaurantes, trenes y autobuses, espectáculos, centros deportivos y spa; en una segunda fase, está alcanzado a todos los sectores económicos (2008: 38 - 39). 
La exaltación del fenómeno low-cost se puede ver en la creación de numerosas empresas en Internet, como Groupon, Let's bonus, o Kviar que ofrecen grandes descuentos para adquirir los servicios antes mencionados, sólo durante un corto periodo de tiempo. Por otra parte, el éxito de Ryanair (Gaggi y Narduzzi, 2006: 43 y 44) radicó en que supo ver que "las necesidades de consumo habían cambiado y que existían decenas y decenas de personas deseosas de viajar a bajo coste". Para ello, eliminó costes e intermediarios innecesarios, organizó su oferta de un modo sencillo y con unos precios bajos. En 2005, un año después de la desregularización del espacio aéreo europeo, "el tráfico de Ryanair subió espectacularmente de tres millones novecientos mil a treinta y cuatro millones de pasajeros". Ryanair no obtiene el mayor volumen de beneficios vendiendo billetes de avión a bajo precio, sino por la venta cruzada de otros servicios como la facturación de maletas; la comisión por pagar con tarjeta de crédito; la comida o bebida dentro del avión; por la publicidad que se puede escuchar en sus aviones antes de despegar y después de aterrizar; o compensando con tarifas más elevadas en temporada alta.

Según Gaggi y Narduzzi (2006), por primera vez en la historia y de manera más amplia, existe un grupo de consumidores "siempre en busca de emplear su tiempo y su dinero de la mejor forma posible" (2006: 44). El cliente o consumidor de bajo coste es un "nómada" que se rige por el "binomio precio - practicidad" para poder repartir su renta entre el mayor número posible de productos y/o servicios posibles. Esta búsqueda constante es posible (Valls et al., 2008) ya que:

El cliente final cada vez está más 'educado' desde un punto de vista tecnológico. Antes de tomar la decisión sobre la compra de un servicio, ya sea el alquiler de coche, una noche de hotel o un espectáculo, el cliente tiene a su alcance numerosas herramientas para comparar precios y condiciones. En otras palabras, el consumidor actual posee un mayor conocimiento del mercado, solicita servicios más específicos y sabe cómo conseguir las mejores tarifas disponibles (2008: $139-140$ ).

A grandes rasgos, los modelos de negocio low-cost (Valls et al., 2008) se basan en dos premisas. La primera es "eliminar o gestionar como opcionales algunas características del producto o servicio que tradicionalmente se habían considerado 'core' y, por tanto, intocables"; y la segunda es:

Romper el modelo en el que el 'up grade' era el referente para el consumidor (por ejemplo, el vehículo más equipado de un determinado modelo de automóvil). La apelación a la 'inteligencia del cliente' es la expresión de esta pérdida de referente al servicio o versión 'mas caros' y su sustitución por la racionalización del precio respecto al valor deseado (2008: 39).

A la sociedad low-cost, se une un nuevo modelo de negocio cimentado en "lo gratis". Se basa (Andersen, 2008) en una "nueva capacidad de reducir costes de bienes y servicios a casi cero" (2008: 29) gracias a los cambios que ha traído la era digital. Ante la sociedad lowcost y donde impera "lo gratis", se alza una "nueva aristocracia, enormemente patrimonializada por la utilización empresarial del conocimiento" que "reclama bienes y 
servicios exclusivos, personalizados" y con un alto valor añadido (Gaggi y Narduzzi, 2006: 48). Este deseo de personalización no sólo caracteriza a una "sociedad de lujo", sino que, tal y como comenta Chris Andersen (2008):

Tendemos a no valorar aquello que tenemos ahora mismo en abundancia. Por ejemplo, la abundancia de café en el trabajo suscita la necesidad de café mucho mejor, por el que estamos dispuestos a pagar mucho. $Y$ también por un producto premium que surja de un mar de productos de consumo barato, desde un alimento artesanal a un agua de diseño (2008: 235).

Según Antonio Lucio (Morgan, 2009), cuando una marca quiere liderar una categoría o mercado tiene que trascender, algo que caracteriza a las marcas de lujo. Una forma de ir más allá es el esfuerzo constante por superar las expectativas. Como afirma Adam Morgan (2009: 94) superar las expectativas supone el punto extremo de diferencia que justifica un posicionamiento emocional, y unos seguidores o embajadores de la marca. Además, se crea una razón de ser para la empresa, un modo de actuar y en el que creer. Querer superar las expectativas de los clientes o consumidores muestra que se cree en el producto, que existe una preocupación real por ofrecer un buen producto o servicio $y$, en el fondo, supone el compromiso de la compañía por trasladar la promesa de la marca bajo una filosofía caracterizada por la excelencia. Las marcas de lujo, además de ser líderes, creen en su producto y/o servicio, de ahí que hayan sido capaces de perdurar, algunas, durante más de un siglo. Si bien ahora las marcas han de reinterpretar los valores para conectar con un consumidor cada vez más indiferente, de ahí que la excelencia (Ricca y Robbins, 2012) pueda ser el vehículo que les ayudará a trascender en una sociedad postmoderna. La excelencia de las marcas de lujo se entiende como una filosofía que trasciende más allá de lo estrictamente empresarial, se entiende como autenticidad, como prueba máxima de la calidad de sus productos o servicios, como marcas generadoras de experiencias únicas, como líderes en la implantación de su visión como marca y en definitiva, como eje básico de diferenciación.

La percepción del precio también influye en las marcas de lujo, Susana Campuzano (2003) distingue tres tipos de lujo en función de los costes y de los márgenes: inaccesible, intermedio y accesible. Precisamente es en los márgenes donde el prestigio, la selectividad y la exclusividad determinan la percepción de la marca para fijar su precio. Por ejemplo, en el lujo inaccesible el factor precio no es determinante en la compra, ya que su diferenciación y superioridad distinguen a la marca de la competencia. En el lujo intermedio el precio tampoco es determinante en la compra porque, la marca y sus valores están asociados a un determinado estatus social, y pesan más en la decisión. En el lujo accesible, la compra se decide en función del binomio calidad-precio, por lo que el precio sí es clave para realizar la compra. 
Figura 1. Fórmula para establecer el precio del lujo.

\begin{tabular}{|l|l|l|}
\hline \multicolumn{1}{|c|}{ LUJO } & \multicolumn{1}{|c|}{ COSTES } & \multicolumn{1}{|c|}{ MARGEN } \\
\hline Inaccesible & $\begin{array}{l}\text { De creación e investigación } \\
\text { De materias primas, de } \\
\text { fabricación y de control de } \\
\text { calidad. } \\
\text { De distribución cautiva y de } \\
\text { comunicación. }\end{array}$ & $\begin{array}{l}\text { De la exclusividad } \\
\text { (valor de creación, } \\
\text { novedad y rareza; } \\
\text { ventajas psicológicas de } \\
\text { distinción; y valor de la } \\
\text { marca) }\end{array}$ \\
\hline Intermedio & $\begin{array}{l}\text { De declinación y creación } \\
\text { De materias primas, de } \\
\text { fabricación y de control de } \\
\text { calidad. } \\
\text { De estudios de competencia y } \\
\text { demanda. } \\
\text { De distribución y de } \\
\text { comunicación. }\end{array}$ & $\begin{array}{l}\text { De selectividad } \\
\text { (valor de diferenciación } \\
\text { del producto; ventajas } \\
\text { psicológicas de } \\
\text { apropiación; y valor de la } \\
\text { marca) }\end{array}$ \\
\hline Accesible & $\begin{array}{l}\text { De declinación y de nuevo } \\
\text { producto. } \\
\text { De materias primas, de } \\
\text { fabricación y de control de } \\
\text { calidad. } \\
\text { De estudios de mercado y de la } \\
\text { competencia } \\
\text { De distribución, de } \\
\text { comunicación y de Marketing. }\end{array}$ & $\begin{array}{l}\text { De prestigio } \\
\text { (ventajas psicológicas de } \\
\text { acceso a una marca) }\end{array}$ \\
\hline
\end{tabular}

Fuente: Reelaborado a partir de El universo del lujo. Una visión global y estratégica para profesionales y amantes del lujo, de Susana Campuzano (2003: 210 - 212).

Se estima que cada vez hay más personas ricas en el mundo. Según el ranking que elabora la revista Forbes, en 2014 se incorporaron 268 nuevas fortunas a su listado de las personas más ricas del mundo. Estados Unidos es el país donde Forbes ha localizado más fortunas, seguido por Asia-Pacífico, Europa, Las Américas y, por último, Europa del Este y África. En el listado de 2014 de Forbes, se puede comprobar que algunas de las personas más ricas del mundo son propietarias de las grandes empresas de bajo coste. Por ejemplo: Amancio Ortega, es el presidente del grupo Inditex; Christy Walton y su familia, son dueños de los supermercados Wal-Mart; Stefan Persson es el dueño de H\&M. Entre las primeras posiciones del listado de Forbes, destacan la primera posición del norteamericano Bill Gates, la segunda del mexicano Carlos Slim, y la tercera del norteamericano Warren Buffett. En la siguiente tabla se puede ver un resumen de las principales posiciones. 
Figura 2. Listado Forbes de 2014 de las personas más ricas.

\begin{tabular}{|l|l|c|c|l|l|}
\hline POSICIÓN & \multicolumn{1}{|c|}{ NOMBRE } & $\begin{array}{c}\text { PATRIMONIO } \\
\text { (en millones de } \\
\text { dólares USD) }\end{array}$ & EDAD & EMPRESA O SECTOR & \multicolumn{1}{|c|}{$\begin{array}{l}\text { PAIS DE } \\
\text { ORIGEN }\end{array}$} \\
\hline 1 & Bill Gates & 77.300 & 58 & Microsoft & Estados Unidos \\
\hline 2 & $\begin{array}{l}\text { Carlos Slim Helu y } \\
\text { familia }\end{array}$ & 71.000 & 74 & Telecomunicaciones & México \\
\hline 3 & Warren Buffett & 65.200 & 83 & Berkshire Hathaway & Estados Unidos \\
\hline 4 & Amancio Ortega & 63.400 & 78 & Inditex & España \\
\hline 5 & Larry Ellison & 50.200 & 69 & Oracle & Estados Unidos \\
\hline 6 & Charles Koch & 40.900 & 78 & Diversificado & Estados Unidos \\
\hline 7 & David Koch & 40.900 & 73 & Diversificado & Estados Unidos \\
\hline 8 & Christy Walton y & 38.800 & 59 & Wal-Mart & Estados Unidos \\
\hline 9 & familia & 36.600 & 80 & Casinos & Estados Unidos \\
\hline 10 & Jim Walton & 36.600 & 64 & Wal-Mart & Estados Unidos \\
\hline
\end{tabular}

Fuente: Adaptado de The World's Billonaires, por Forbes (2014).

Ante los datos de aumento de fortunas en el mundo y como afirman Gaggi y Narduzzi (2006), se inicia una nueva era caracterizada por la división de la sociedad en cuatro grupos muy diferenciados en cuanto su poder adquisitivo y comportamientos de consumo. Los grupos son una nueva aristocracia, una élite de tecnócratas, la sociedad masificada y una clase proletarizada.

[En primer lugar se encuentra] una [nueva] aristocracia muy patrimonializada y acaudalada, capaz de asegurar consumos significativos de bienes; son los vencedores en la ruleta de la innovación capitalista que ha vuelto a pagar con sumas astronómicas la apuesta, entendida como una idea transformadora en producto o servicio original, concebida para dar vida a empresas de éxito. [En segundo lugar, se distingue] una élite bastante numerosa de tecnócratas del conocimiento con rentas medio-alta y notable capacidad para consumir lo que ahora ya ofrece a gran escala la industria de los países avanzados; los componentes de esta élite alternan consumos personalizados con un valor añadido con adquisiciones decididas según la conveniencia del precio requerido; raramente ascienden al olimpo de los millonarios mientras que con mayor facilidad pueden encontrarse, en el curso de la vida, degradándose en la masa. [El tercer grupo está representado por] una sociedad masificada de renta mediobaja pero a la que la industria de bajo coste garantiza el acceso a bienes y servicios en otro tiempo reservados a clases más acomodadas: el elemento de decoración diseñado por el elitista de genio, pero a precios de Ikea; los 'autobuses del cielo' que permiten, a un precio asequible, eliminar los rigores del invierno con breves estancias en climas templados. Pero también los cruceros en naves nodrizas que, si bien vistas desde fuera parecen rascacielos flotantes, resultan en realidad cómodas y ofrecen multitud de servicios y una gran cantidad de diversiones. Es lo que podríamos llamar 'Ryanair society' o sociedad de bajo coste. [En último lugar, los autores distinguen a] una clase 'proletarizada' o con escaso poder adquisitivito (desde los obreros hasta los pensionistas sin rentas complementarias, pasando por los profesores y funcionarios con una familia a su cargo y que cobran el salario mínimo), que consumirá bienes de primera necesidad, sustituirá el coche por el transporte público y vivirá de servicios sociales esenciales, cada vez más próxima a modelos sociales de 'Tercer Mundo' emergente (2006: 9 y 10).

Por su parte, la industria del lujo cambió drásticamente para aprovechar los cambios y las oportunidades que surgían en el mercado. La industria del lujo, tras identificar los cambios 
en sus hábitos de consumo de los consumidores, comenzó a democratizar parte de sus marcas para asegurar sus ingresos. En este sentido, Lipovetsky (2004) afirma que "el lujo ha estallado, ya no cabe hablar de un lujo, sino de lujos, a varios niveles, para públicos diversos" (2004: 15). Según este autor, se trata de hacer "accesible lo inaccesible", por esta razón, Lipovetsky distingue dos tipos de lujo: el lujo "extremadamente elitista" y el lujo de la "democratización de masas". Estos dos tipos de lujo coinciden con las dos estrategias empresariales imperantes en el sector del lujo. Lipovetsky menciona que "coexisten dos tendencias: una banaliza el acceso al lujo y lo desmitifica, la otra reproduce el poder de ensueño y de atracción mediante las políticas de precios y de imagen" (2004: 16). Dicha división coincide con la afirmación de Ricca y Robbins (2012) sobre la pérdida del significado original del lujo. Prueba de ello es la utilización, por un lado, de superlativos como "hyperluxury, super-luxury, ultra-luxury (y) high-luxury" para distinguir a las verdaderas marcas de lujo. O el uso, por otro lado, de términos como "premium, super-premium, high-end (o) exclusive" (2012: XI) de algunas marcas de gran consumo que desean crear una atmósfera más cercana a los productos de lujo. El interés por democratizar las marcas de lujo radica, según describe Dana Thomas (2007: 9 - 13), en el potencial que los grandes magnates financieros vieron en este mercado. Para ello, estos empresarios diseñaron una estrategia para "democratizar" el lujo con el objetivo de llegar al gran público y por lo tanto, vender y ganar más dinero. Tal y como describe Thomas, la estrategia para convertir el lujo en unos bienes y servicios más accesibles, se realizó en dos fases: en primer lugar, hubo una inversión desmesurada en publicitar las marcas de lujo y después, se produjo el lanzamiento de productos de entrada accesibles.

En la primera fase de la democratización del lujo, las marcas de lujo comunicaron, por un lado, el legado histórico de la marca y su artesanía de forma exagerada, para así legitimar a los productos con un estatus lujoso. Por otro lado, se lanzaron campañas impactantes para incrementar su notoriedad y reconocimiento por pate del gran público. Por ejemplo, el anuncio creado por el diseñador Tom Ford de la fragancia de Opium de Yves Saint Laurent en las navidades del año 2000 fue retirado en países como Reino Unido por considerarlo "indecente". En dicho anuncio aparecía la modelo Sophie Dahl, conocida por alejarse del canon de delgadez y por usar una talla 44, desnuda sobre un fondo negro en una posición erótica. Thomas (2007) resalta que los diseñadores se vieron obligados a convertir sus desfiles en shows extravagantes y muy llamativos para captar la atención de los medios de comunicación de todo el mundo. Las marcas de lujo también comenzaron a vestir a personajes famosos y actores que asistían a eventos mediáticos como desfiles, entregas de premios, estrenos, inauguraciones o fiestas. Muchas otras marcas de lujo siguieron el ejemplo al patrocinar eventos de alto nivel como venía haciendo Louis Vuitton con la America's Cu: Por su parte, Rolex, además de patrocinar diversas competiciones de golf en Estados Unidos y en Europa, creó su propio torneo golf. Una vez el público medio ya conocía las principales de marcas de lujo, Thomas afirma que fue el momento para lanzar accesorios, colonias y maquillaje con un precio que estaba al alcance de muchos más bolsillos. Por ejemplo, hoy en día, un pintalabios de Chanel cuesta unos 25 euros y un perfume de $125 \mathrm{ml}$. 
de Dolce \& Gabbana unos 60 euros. En paralelo, las grandes marcas de lujo aplicaron el modelo de integración vertical (Thomas, 2007) diseñado por Henry Racamier en 1977 cuando asumió la presidencia de Louis Vuitton. La innovación de este modelo suponía eliminar las licencias y franquicias para distribuir los productos de la compañía, en especial, en el extranjero y así abrir tiendas propias donde se pudiesen vender todos los productos. Esta integración le permitió a Louis Vuitton, al poco tiempo, tener beneficios de un 40\%, mientras que sus competidores de la época obtenían entre un 15 y un 25\%. Las tiendas propias se abrieron a modo de flagship en las ciudades más cosmopolita del mundo como Nueva York, Los Ángeles, Londres, Milán o París. De ahí, se copió el modelo de tienda y de experiencia exportándolo a otras ciudades. A esto se sumó la apertura de outlets; la apuesta definitiva por la presencia de estas marcas en el comercio aeroportuario de duty free; y el lanzamiento de páginas de Internet para comprar productos de lujo. Además, el sector del lujo se ha convertido en uno de los grandes captadores de talento empresarial. Como muestra, destaca la trayectoria del propio Bernard Arnault (Cortázar, 2007), presidente y CEO del Grupo LVMH, ingeniero de formación y que hizo su primera fortuna dedicándose a las transacciones inmobiliarias.

Por su parte, las marcas low-cost han conseguido democratizar el lujo masificando las marcas premium. La consecuencia ha sido la radicalización del consumo (Andersen, 2008) y la aparición de fenómenos como el que el diseñador Isaac Mizrahi define como "bipolar shopping disorder [desorden de compra bipolar]" (Thomas, 2007: 318). Este fenómeno explicaría el éxito de las colecciones que diseñadores como Karl Lagerfeld, Jimmy Choo o Lanvin hicieron para H\&M. Según explica Lagerfeld:

We live in a time when expensive and inexpensive -not cheap, I hate that Word- can live very well together. It's the first time in fashion this has happened [Vivimos en un tiempo de lo caro y no caro -no barato, odio esa palabra- todos podemos vivir juntos muy bien. Esta es la primera vez que ha sucedido en la moda]" (Thomas, 2007: 318 - 3 19).

Marcas como Calvin Klein, los relojes Swatch o el agua Evian, se han establecido como referente en el modelo de gestión de las marcas low-cost con productos básicos más accesibles para el gran público. En cambio, otras marcas del sector textil como H\&M, Zara o Gap, han sabido envolverse bajo un aire premium en sus flagship stores. Destaca el caso de la marca británica de moda Topshop, considerada como una cadena "cheap and chic" [barata y de moda] (Grant, 2006: 207). Durante los últimos años, Topshop ha incorporado recursos de las grandes marcas de lujo como la colaboración con diseñadores de primera línea, la exhibición de sus diseños en el certamen de moda London's Fashion Week o la participación en eventos de acción social para ayudar a los más desfavorecidos. Lo que más llama la atención es su nuevo servicio gratuito de "personal shopping" [asistente de compras] (2006: 208) en el cual un estilista ayuda a las clientas a elegir la ropa que mejor les sienta o según la ocasión; les lleva la ropa al probador en función de unas directrices de la clienta; les da acceso a un lugar VIP de la tienda; les envuelve las prendas para regalo; hace que no tengan que esperar para pagar; o incluso pueden llevar la ropa al lugar donde el cliente desee. Este 
servicio no requiere un mínimo de gasto, pero el éxito es tal, que por cada cita con el personal shopper, la compra media es de unas 250 libras. En este sentido, Marc Gobé (2005) afirma que los consumidores que pertenecen a lo que se conoce como "la generación X" están potenciando el consumo de marcas premium y con un cierto aire cool [moderno], como símbolo de status. En palabras de Gobé, "la generación X se está convirtiendo en la generación $<<\mid u^{\prime} X^{\prime}$ e $>>$ a medida que va rompiendo las reglas y definiendo una nueva economía. Ganan más dinero para demostrar que ahora pueden permitírselo" (2005: 20). A este fenómeno se suma lo que sucede en muchas de las calles donde tradicionalmente sólo había tiendas de marcas de lujo. Por ejemplo, si se pasea por la Quinta Avenida de Nueva York, se puede ver lo que Dana Thomas (2007:265) define como la "integración de tiendas" de marcas de lujo y de marcas low-cost, algo que se ha convertido en un reclamo turístico. Marcas como Zara, Gap o Abercrombie \& Fitch conviven al lado de Louis Vuitton, Gucci, De Beers o Bottega Veneta.

\section{Conclusiones: los retos para las marcas en una sociedad bipolar}

Nos encontramos en una nueva era donde los hábitos de consumo se han polarizado. Los cambios en la percepción del factor precio de los consumidores han sido determinantes. Por ello, se debe hacer un esfuerzo mayor en comunicar constantemente el posicionamiento de la marca y su propuesta de valor para reforzar los atributos tangibles e intangibles de la marca. De hecho, si se dibujase un reloj de arena, en los extremos se encontrarían el lujo y el low-cost, por eso, es palpable cómo muchas marcas que se encuentran en el centro intentan sobrevivir en el mercado virando su estrategia hacia lo premium. Dicha situación ha sido provocada, en parte, por la ampliación del nicho de mercado de las marcas de lujo para aumentar sus beneficios. Además, hay muchos autores y diseñadores que opinan que el lujo roza más la percepción que la realidad de la calidad y excelencia del producto o servicio en cuestión, argumento que refuerza la polarización del consumo. Dicha percepción está también unida al valor percibido del producto o servicio de una marca de lujo que debe estar íntimamente relacionada con la excelencia.

Las empresas tratan de adaptar su estrategia de negocio para mejorar sus resultados en un mercado muy polarizado donde los consumidores tienen como aliada a la tecnología para tener un mayor conocimiento del mercado y de las diferentes alternativas que les permita demandar productos y servicios más concretos a un mejor precio. De ahí que, en relación a los efectos de las marcas low-cost y de las marcas de lujo en los hábitos de consumo en los consumidores, se observa una radicalización del consumo. Por una parte, se encuentra a un consumidor que se rige por el binomio precio - practicidad para sacar mayor partido a su 
renta; y por otra parte, existe un consumidor que desea consumir productos y servicios muy exclusivos que le permitan diferenciarse del resto de consumidores de marcas low-cost.

De los planteamientos estratégicos de las compañías de lujo y low-cost se pueden determinar una serie de similitudes y diferencias. Los modelos low-cost estructuran su oferta de manera simple para quedarse con la esencia del negocio y así eliminar los costes innecesarios que justifiquen apelar a la inteligencia del cliente para elegir su marca. Por su parte, las compañías de lujo han conseguido grandes beneficios aplicando el modelo de integración vertical que elimina licencias y franquicias para recuperar la distribución de sus propios productos a nivel mundial. La apertura de flagships ha permitido proyectar la marca de un modo coherente en los distintos puntos de venta y exportar el modelo a otras categorías. De hecho, las marcas low-cost han conseguido democratizar el lujo abriendo sus propias flagship y envolviéndolas bajo un aire premium. Además, tanto las marcas low-cost como las marcas de lujo, tratan de aportar un valor añadido al consumidor en términos de valor, haciendo así que la competencia sea irrelevante. Ambas también ponen en el centro de su estrategia al consumidor, de él depende los atributos que se dan al producto o servicio para determinar después los precios. De hecho, las marcas de lujo tratan de ensalzar y personalizar aquello que más valora el consumidor para que la marca sea más relevante y aumentar así sus beneficios por el margen de cada producto y/o servicio; mientras que las marcas low-cost potencian lo más relevante del producto o servicio para eliminar costes superfluos y poder aumentar sus beneficios por el volumen de ventas.

La continuidad de este artículo vendría de la mano de un seguimiento de la polarización de los hábitos de consumo y de las nuevas consecuencias que pudiera tener. Además, sería interesante estudiar si, una vez superada la recesión económica, los cambios en la percepción del precio se mantienen y si las marcas de lujo y las de low-cost siguen compartiendo enfoques estratégicos para asegurar su rentabilidad. 


\section{REFERENCIAS BIBLIOGRÁFICAS}

20

MINUTOS (2010): "Anuncios que crean polémica", en http://listas.20minutos.es/lista/anuncios-que-crean-polemica-197824/ (consultado: 06/05/2014)

ABC (2007) "El Dacia Logan de Renault, creado para los mercados emergentes, uno de los coches más demandados", en http://www.abc.es/hemeroteca/historico-14-01-2007/abc/Economia/eldacia-logan-de-renault-creado-para-los-mercados-emergentes-uno-de-loscoches-mas-demandados_153908751270.html\#

ANDERSEN, Chris (2008): Gratis. El futuro de un precio radical. Barcelona, Ediciones Urano.

Bellaiche, Jean-Marc, Mei-Pochtler, Antonella, Hanisch, Dorit (2010): "The New World of Luxury. Caught between growing momentum and lasting change". The Boston Consulting Group, en http://www.bcg.de/documents/file67444.pdf (consultado: 10/06/2014)

BenITO, María (2014): "La industria del lujo verá un incremento de salidas a bolsa y fusiones de firmas en 2014", El Confidencial, en http://www.elconfidencial.com/empresas/2014-01-19/la-industria-del-lujovera-un-incremento-de-salidas-a-bolsa-y-fusiones-de-firmas-en2014_77414/ (consultado: 07/09/2014)

CAMPuZANo, Susana (2003): El universo del lujo. Una visión global y estratégica para profesionales y amantes del lujo. Madrid, McGrawHill.

CORTÁZAR, Beatriz (2007): "Bernard Arnault. El emperador del lujo", ABC, en http://www.abc.es/hemeroteca/historico-09-08-2007/abc/Gente/bernardarnault-el-emperador-del-lujo_164317438281.html (consultado: 07/09/2014)

FORBES (2014): "Inside the 2014 billionaires list: facts and figures", en http://www.forbes.com/sites/luisakroll/2014/03/03/inside-the-2014-forbesbillionaires-list-facts-and-figures/ (consultado: 10/05/2014)

- (2014): "The 85 richest people in the world have as much wealth as the 3.5 billion poorest", en http://www.forbes.com/sites/laurashin/2014/01/23/the85-richest-people-in-the-world-have-as-much-wealth-as-the-3-5-billionpoorest/ (consultado: 10/05/2014)

- (2014): "The world's billonaires", en http://www.forbes.com/billionaires/list/\#tab:overall (consultado: $10 / 05 / 2014)$ 
GaGGI, Massimo, NARDUZzI, Edoardo (2006): "El fin de la clase media y el nacimiento de la sociedad de bajo coste". Madrid, Lengua de trapo.

GoBÉ, Marc (2005): "Branding emocional. El nuevo paradigma para conectar las marcas emocionalmente con las persones". Barcelona, Divine Egg Publicaciones.

GRANT, John (2006): "The brand innovation manifesto. How to build brands, redefine markets and defy conventions". Sussex, Wiley.

HoffMANN, Jonas, COSTE-MANIERE, Ivan (2012): "Luxury Strategy in action". Londres, Palgrave Macmillan.

HolA. "Tom Ford relanza los perfumes de Yves Saint Laurent", en http://www.hola.com/belleza/fragancias/2003070247335/belleza/fragancias /fraganciastom/ford/perfumes/(consultado: 10/06/2014)

- (2009). "Una Alta Costura comedida, pero llena de ingenio, llega a su fin", en http://www.hola.com/noticias-de-actualidad/29-01-2009/62927/ (consultado: 12/07/2014)

LeNT, Robin, Geneviève, Tour (2009): Selling Luxury. Nueva Yersey, Wiley.

LIPOVETSKY, Gilles, Roux, Elyette (2004): El lujo eterno. De la era de lo sagrado al tiempo de las marcas. Barcelona, Anagrama.

MORGAN, Adam (2009): Eating the big fish. How challenger brands can compete against brand leaders. Nueva Yersey, Wiley.

NACIONES UNIDAS. "Las cifras sobre la pobreza". Programa para el Desarrollo, en http://www.teamstoendpoverty.org/wq_pages/es/visages/chiffres.php (consultado: 05/06/2014)

NUÑEZ, Ana (2010): "Lanvin: nueva colección exclusiva para H\&M, a la venta el 23 de noviembre", Belleza en http://www.bellezapura.com/2010/10/25/lanvin-nueva-coleccion-exclusivapara-hm-a-la-venta-el-23-de-noviembre/ (consultado: 21/07/2014)

RiccA, Manfredi, RoBbins, Rebecca (2012): "Meta-luxury. Brands and the culture of excellence". Londres, Palgrave Macmillan.

SICARD, Marie-Claude (2007): "Lujo, mentiras y marketing". Barcelona, Ediciones Gustavo Gili.

Thomas, Dana (2007): "Deluxe: How luxury lost its luster". Nueva York, Penguin Books.

TUNGATE, marc (2005): "Fashion brands. Branding style from Armani to Zara". Londres, Kogan Page.

- (2009). "Luxury world. The past, present and future of luxury brands". Londres, Kogan Page. 
VALLS, Josep (2008): "Fenómeno low-cost. El impacto en el factor precio". Barcelona, Deusto.

[Recibido: 22 de septiembre de 2014. Aceptado: 2 de febrero de 2016] 